\title{
Bronchopulmonary Kaposi's sarcoma in patients with AIDS
}

\author{
R F Miller, M C Tomlinson, C P Cottrill, J J Donald, M F Spittle, S J G Semple
}

\begin{abstract}
Background Kaposi's sarcoma in HIV antibody positive patients may affect the lungs. This study describes the presentation, chest radiographic appearances, and pulmonary function test abnormalities in patients with AIDS who had tracheobronchial Kaposi's sarcoma.
\end{abstract}

Methods and results Twenty nine (8\%) of 361 consecutive HIV antibody positive patients undergoing bronchoscopy for respiratory symptoms had tracheobronchial Kaposi's sarcoma. Eight patients had intercurrent infections and one had previously received chemotherapy for cutaneous Kaposi's sarcoma; these patients were excluded. Seven of the remaining 20 patients had localised Kaposi's sarcoma (lesions confined to the trachea or the subsegments of one lobe) and 13 had widespread Kaposi's sarcoma (affecting the trachea and one lobe or the subsegments of more than one lobe); 19 patients also had cutaneous and palatal Kaposi's sarcoma. Seven patients, four with widespread disease, had a normal radiograph. All patients had reduced transfer factor (TLCO) and transfer coefficient (KCO) but only those with widespread disease had reductions in forced expiratory volume in one second $\left(F E V_{1}\right)$, forced vital capacity (FVC), and peak expiratory flow (PEF). Follow up pulmonary function testing in seven patients (median three months later) showed further reductions in TLCO. All four patients who received no treatment had progressive radiographic abnormalities; bronchoscopy in two patients showed progressive tracheobronchial disease, and two patients had further reductions in FEV 1 and FVC. In three patients treated with chemotherapy palliation of symptoms was achieved but two had further reductions in $F E V$, and FVC and the radiograph deteriorated. Bronchoscopy showed regression of disease in only one patient.

Conclusion Pulmonary Kaposi's sarcoma produces abnormalities of TLCO even in patients with localised disease; airflow obstruction may occur in patients with widespread disease. Bronchoscopic reassessment of the extent of disease may not accurately reflect response to chemotherapy.

(Thorax 1992;47:721-725)
Kaposi's sarcoma occurs in up to a quarter of patients with the acquired immunodeficiency syndrome (AIDS) and is particularly common in homosexual and bisexual men. ${ }^{1}$ The most common site is the skin, where multiple lesions occur. Within the chest Kaposi's sarcoma may occur in the tracheobronchial tree, lung parenchyma, mediastinal lymph nodes, or visceral pleura. ${ }^{2}$ The exact incidence of intrathoracic Kaposi's sarcoma in patients with AIDS is unknown. In clinical studies pulmonary Kaposi's sarcoma has been reported in 3-13\% of patients with AIDS $^{23}$ and in up to $32 \%$ of patients with AIDS who had cutaneous Kaposi's sarcoma. ${ }^{245}$ In contrast, bronchopulmonary Kaposi's sarcoma was found at necropsy in $47 \%$ of patients with AIDS who had cutaneous Kaposi's sarcoma. ${ }^{6}$

The diagnosis of pulmonary Kaposi's sarcoma may be difficult to make during life. Presenting symptoms and chest radiographic appearances are non-specific and non-invasive investigations, including exercise testing and arterial blood gas estimation, do not distinguish bronchopulmonary Kaposi's sarcoma from other causes of lung disease in AIDS. Tracheobronchial lesions may not be seen at bronchoscopy ${ }^{7}$ (in the presence of parenchymal tumour) and parenchymal lesions are often patchy, and so may be missed by transbronchial biopsy. ${ }^{346-8}$ Histological diagnosis from bronchial or transbronchial biopsy specimens is often difficult because of the submucous distribution of the endobronchial tumour and because the lesions of Kaposi's sarcoma rarely show features of malignancy ${ }^{7}$; crush artefact, haemorrhage, and granulation tissue may all mimic Kaposi's sarcoma. Diagnosis is often made by visual identification of lesions at bronchoscopy. ${ }^{46-8}$ Tracheobronchial lesions have a characteristic appearance: they are typically red or violaceous, flat or raised discrete plaques and they appear similar to cutaneous Kaposi's sarcoma. In this study we describe the clinical features, chest radiographic appearances, and abnormalities of lung function in patients with AIDS in whom tracheobronchial Kaposi's sarcoma had been diagnosed at bronchoscopy.

\section{Patients and methods}

Three hundred and sixty one consecutive HIV-1 antibody positive patients underwent fibreoptic bronchoscopy for investigation of respiratory episodes over the four years January 1988 to December 1991. Before bronchoscopy in each patient the presence and duration 
of respiratory symptoms was noted and a smoking history obtained. The presence of cutaneous and palatal Kaposi's sarcoma was also recorded, as was a past history of Pneumocystis carinii pneumonia or other HIV related respiratory problems.

At bronchoscopy (all bronchoscopies were performed by RFM) the tracheobronchial tree was carefully examined for the presence of Kaposi's sarcoma. Tracheobronchial sarcoma was categorised as localised if the lesions were seen only in the segmental bronchi of a single lobe or on the wall of the trachea, or widespread if both a single lobe and the trachea were affected or if the segmental bronchi of two or more lobes were affected. Bronchoalveolar lavage ${ }^{9}$ was performed from the lobe most affected by radiographic shadowing or, in patients with diffuse radiographic abnormalities, from the right middle lobe. Lavage fluid was stained with Grocott's silver stain, auramine, and Gram stain to identify Pneumocystis carinii, acid and alcohol fast bacilli, and bacteria respectively and cultured for viruses, bacteria, mycobacteria, and fungi. ${ }^{9}$ To detect occult alveolar haemorrhage, in some cases lavage fluid was stained with Perl's iron stain for identification of haemosiderin laden macrophages. ${ }^{10}$

Biopsies of Kaposi's sarcoma lesions were not performed, because of the low diagnostic yield and the risk of haemorrhage. ${ }^{346-8}$ Diagnosis was made on the basis of characteristic appearances; in some patients this was confirmed subsequently after necropsy. All patients had a chest radiograph, which was reported retrospectively by a radiologist (JJD) who was blind to the diagnosis.

Pulmonary function tests were carried out either before or 72 or more hours after bronchoscopy. The following were measured: forced expiratory volume in one second $\left(F E V_{1}\right)$, forced vital capacity (FVC), peak expiratory flow (PEF). Transfer factor for carbon monoxide (TLCO) and transfer coefficient (Kco) were measured by the single breath helium dilution method. All measurements were made with an Autolink system (PK Morgan Ltd, Chatham). Values for TLCo and Kco were corrected for temperature and the patient's haemoglobin concentration.

Lung function results, duration of symptoms and of cutaneous Kaposi's sarcoma, and survival after diagnosis were compared in those with localised and with widespread Kaposi's sarcoma by means of Wilcoxon's rank sum test; a $p$ value of less than 0.05 was taken to be significant.

\section{Results}

Twenty nine men out of $361(8 \%)$ consecutive HIV-1 antibody positive patients who underwent bronchoscopy had tracheobronchial lesions of Kaposi's sarcoma. All were white; 28 were homosexual or bisexual and one heterosexual with no apparent risk factors for HIV infection; none were currently using intravenous drugs or had previously done so. Their mean age was 40 (range 29-55) years. In seven patients infective pathogens were identified in bronchoalveolar lavage fluid. Of three patients with localised Kaposi's sarcoma, two had Penumocystis carinii and one had Staphylococcus aureus and acid and alcohol fast bacilli (subsequently identified as Mycobacterium avium-intracellulare). Of four patients with widespread Kaposi's sarcoma, two had $S$ aureus, one had $P$ carinii, and one other had acid and alcohol fast bacilli (subsequently identified as Mycobacterium tuberculosis). Another patient with widespread Kaposi's sarcoma had intercurrent Escherichia coli septicaemia arising from a Hickman line infection. Data from these eight patients are excluded as interpretation of symptoms, chest radiographic appearances, and pulmonary function data ${ }^{11}$ in the presence of intercurrent infection would be unreliable. One other patient with localised Kaposi's sarcoma had previously received chemotherapy for cutaneous Kaposi's sarcoma and was also excluded. None of the remaining 20 patients with tracheobronchial Kaposi's sarcoma, who were free from infection, presented with new respiratory symptoms within six weeks.

\section{BRONCHOSCOPIC APPEARANCES}

In seven of the 20 patients the tracheobronchial Kaposi's sarcoma was localised and in 13 patients the lesions were widespread.

\section{SYMPTOMS AND SIGNS}

All 20 patients had cough and breathlessness, but no patient had chest pain or haemoptysis. The overall duration of symptoms ranged from one to 18 (median 4) weeks. In patients with widespread tracheobronchial Kaposi's sarcoma the duration of symptoms ranged from 1-18 (median $=4$ ) weeks and in those with localised disease ranged from two to 12 (median 4) weeks (NS). Seventeen patients were cigarette smokers. Of seven patients with localised Kaposi's sarcoma, five had no previous respiratory episodes and two had prior pneumocystis pneumonia (three and 13 months previously). Of the 13 patients with widespread disease, five had no prior respiratory history, seven had pneumocystis pneumonia (median 14 (range 2-48) months previously) and one patient had had Streptococcus pneumoniae pneumonia eight months previously.

Table 1 Results of lung function tests (mean (SD) \% predicted ${ }^{\star}$ ) in patients with tracheobronchial Kaposi's sarcoma

\begin{tabular}{lllc}
\hline & \multicolumn{2}{l}{ Kaposi's sarcoma } & \\
\cline { 2 - 3 } & $\begin{array}{l}\text { Localised } \\
(n=7)\end{array}$ & $\begin{array}{l}\text { Widespread } \\
(n=13)\end{array}$ & $p$ \\
\hline FEV & $88(13)$ & $61(19)$ & $<0.05$ \\
FVC & $89(9)$ & $70(21)$ & $<0.05$ \\
PEF & $82(22)$ & $59(16)$ & $<0.02$ \\
TLCO & $61(13)$ & $72(14)$ & NS \\
KCO & $53(9)$ & $66(13)$ & NS \\
\hline
\end{tabular}

«For white males.

$\mathrm{FEV}_{1}$ - forced expiratory volume in one second; FVCforced vital capacity; PEF-peak expiratory flow; TLCotransfer factor for carbon monoxide; Kco-transfer coefficient. 
Table 2 Chest radiograph appearances in patients with tracheobronchial Kaposi's sarcoma

\begin{tabular}{|c|c|c|}
\hline \multirow[b]{2}{*}{ Radiographic appearance } & \multicolumn{2}{|c|}{ Kaposi's sarcoma } \\
\hline & $\begin{array}{l}\text { Localised } \\
(n=7)\end{array}$ & $\begin{array}{l}\text { Widespread } \\
(n=13)\end{array}$ \\
\hline Normal & 3 & 4 \\
\hline Perihilar infiltrates & - & $\begin{array}{l}3 \text { (2 also had hilar lymph node enlargement and one of these a } \\
\text { pleural effusion) }\end{array}$ \\
\hline Bilateral interstitial shadows & 3 & $\begin{array}{l}1 \text { (also had bilateral pleural effusions and right middle and lower } \\
\text { lobe collapse) }\end{array}$ \\
\hline Bilateral coarse reticulonodular shadows & 1 & 4 ( 1 also had hilar lymph node enlargement and a pleural effusion) \\
\hline Focal or lobar consolidation & - & 1 (also had bilateral pleural effusions) \\
\hline
\end{tabular}

Nineteen of the 20 patients had both cutaneous and palatal Kaposi's sarcoma; the patient who was the exception had localised endobronchial disease. The time from diagnosis of cutaneous Kaposi's sarcoma to diagnosis of tracheobronchial Kaposi's sarcoma ranged from two to 37 months (median 10 months) in those with widespread disease and from three to 28 months (median 10 months) in those with localised disease (NS). In 10 patients (four with localised tracheobronchial Kaposi's sarcoma) Perl's staining of bronchoalveolar lavage fluid was carried out. In eight patients (including all six with widespread disease) haemosiderin laden macrophages were identified, providing evidence of occult alveolar haemorrhage.

\section{RESULTS OF PULMONARY FUNCTION TESTS}

$\mathrm{FEV}_{1}, \mathrm{FVC}$, and PEF were normal in patients who had localised tracheobronchial Kaposi's sarcoma (table 1) but were reduced in patients with widespread disease $(p<0.05)$. TLCo and Kco were reduced in both groups of patients. There was no correlation between TLCO or $\mathrm{KCO}$ and smoking history or the presence or absence of occult alveolar haemorrhage.

\section{CHEST RADIOGRAPHIC APPEARANCES}

Seven patients, four with widespread tracheobronchial Kaposi's sarcoma, had normal chest radiographs. In the remainder several chest radiographic abnormalities were seen (table 2 ). Severe abnormalities occurred more frequently in patients with widespread tracheobronchial Kaposi's sarcoma, but there was no correlation between the bronchoscopic extent of Kaposi's sarcoma and the degree of radiographic abnormality.

\section{TREATMENT AND SURVIVAL}

The choice of treatment was based on consideration of many factors, including extent of cutaneous and pulmonary disease, intercurrent HIV related problems and patient preferences. Eleven patients, three with localised disease had no treatment. Nine patients received chemotherapy, three with epirubicin $10 \mathrm{mg}$ IV once weekly, five with vincristine $2 \mathrm{mg}$ IV and bleomycin $30 \mathrm{mg}$ given intravenously once every three weeks (one patient also received radiotherapy to the mediastinum) and one, who was neutropenic, received bleomycin and radiotherapy to the mediastinum. Palliation of symptoms was achieved in all patients. Median survival for the whole group was 18 weeks (range 1-78 weeks).
RESULTS OF REPEAT PULMONARY FUNCTION TESTS Follow up tests were performed in seven patients (of whom five also had repeat rebronchoscopy) two to six (median 3) months after the original investigations; at bronchoscopy no infections were identified. Four patients who received no treatment all had progressive cutaneous Kaposi's sarcoma and bronchoscopy in three showed progressive tracheobronchial disease. One patient had a stable chest radiograph; progressive abnormalities were seen in the other three. All four patients had further reductions in TLCO and in addition two also had further reductions in $\mathrm{FEV}_{1}$ and FVC. The three patients who received chemotherapy, despite palliation of symptoms, had further reductions in TLCO; FEV ${ }_{1}$ and FVC fell further in two patients. In one of these patients bronchoscopy showed regression of tracheobronchial disease and in the other the disease had progressed; the third patient showed no change.

\section{HISTOLOGICAL DIAGNOSIS}

Formal histological diagnosis was made in life in one patient by open lung biopsy three months after bronchoscopic diagnosis and at necropsy in a further seven patients. All seven patients had tracheobronchial lesions and lesions of the visceral pleura. Six also had parenchymal lesions and three had Kaposi's sarcoma of hilar and mediastinal lymph nodes.

\section{Discussion}

At presentation the duration of symptoms of cough and dyspnoea in our patients was variable and did not correlate with the extent of tracheobronchial Kaposi's sarcoma as assessed by bronchoscopy, the radiographic abnormalities, or the results of the lung function tests. In contrast to those in other studies, none of our patients had chest pain or haemoptysis. ${ }^{67}$ We did not carry out bronchial or transbronchial biopsy, because of the low diagnostic yield and the attendant risks of haemorrhage..$^{6-8}$ All but one patient had cutaneous and palatal Kaposi's sarcoma, which supported the diagnosis of tracheobronchial Kaposi's sarcoma, and a tissue diagnosis was made subsequently in eight of the patients who had this diagnosed at bronchoscopy. Occult alveolar haemorrhage was found in eight patients; this finding is thought to be specific for pulmonary Kaposi's sarcoma. In one study of HIV positive patients it was found in six of nine patients with 
pulmonary Kaposi's sarcoma and in none of 75 patients with other diagnoses, including tuberculosis. ${ }^{12}$ Others have reported that occult alveolar haemorrhage is non-specific in HIV positive patients with respiratory symptoms, as in addition to occurring in patients with pulmonary Kaposi's sarcoma it was also found in patients with pneumocystis pneumonia, bacterial pneumonia, and mycobacterial infection. ${ }^{10}$ Seven of our patients, including four with widespread tracheobronchial disease, had normal chest radiographs; this suggests that bronchoscopic assessment of the extent of tracheobronchial Kaposi's sarcoma does not accurately reflect the extent of parenchymal lesions.

There are few data on abnormalities of pulmonary function in HIV positive patients with pulmonary Kaposi's sarcoma. In one study 11 of 12 patients with pulmonary Kaposi's sarcoma, tested before chemotherapy, had a TLCo below $80 \%$ of the predicted value, the mean TLco being $64 \%$ (range 48-84\%). All but one patient had multiple lesions of Kaposi's sarcoma seen at bronchoscopy, but the extent of the disease was not quantified. ${ }^{5}$ In another study eight of eleven patients with pulmonary Kaposi's sarcoma (confirmed at necropsy) had visible tracheobronchial lesions at bronchoscopy. All patients had reductions in TLco but FVC was normal ( $>80 \%$ predicted) in eight patients. Six patients had evidence of airflow obstruction, which did not correlate with smoking history. Four of these six patients had tracheal or endobronchial Kaposi's sarcoma. Four other patients, who also had tracheal or bronchial Kaposi's sarcoma, did not have an obstructive pattern of results in pulmonary function tests. ${ }^{6}$ In this study eight patients had infections as well as Kaposi's sarcoma, $P$ carinii in six and Haemophilis influenzae in one, which makes interpretation of the data on lung function unreliable. ${ }^{6}$ In a study of 16 patients with pulmonary Kaposi's sarcoma who had lesions visible at bronchoscopy TLCO was below $80 \%$ of predicted in three of nine patients tested (mean (SD) $78 \%$ (14.5\%) predicted); the FVC was $73 \%(15.4 \%)$ predicted. In the same study 11 of 15 other patients with pulmonary Kaposi's sarcoma and coinfections, including pneumocystis pneumonia and mycobacterial infection, underwent pulmonary function testing. All these patients had a TLCo below $80 \%$ predicted (mean (SD) $58 \%(9.8 \%)$ ); FVC was $66 \%(21 \%)$ predicted. ${ }^{13}$ In the single case of pulmonary Kaposi's sarcoma reported by Shaw and colleagues TLCo was $56 \%$ predicted, FVC $60 \%$, and PEF $37 \%$; the extent of sarcoma in the airways was not recorded."

In our study we excluded patients with concomitant pulmonary infection or septicaemia; in addition to making interpretation of symptoms and chest radiographs difficult pneumocystis pneumonia and other infections in HIV positive patients may cause substantial reductions in TLCo and FVC and so make interpretation of lung function data unreliable. ${ }^{11}$ In addition, the patient who received prior chemotherapy was excluded from analysis as assessment of the extent of tracheobronchial diseases was not possible, and effects of bleomycin on pulmonary function could not be excluded. Reductions in spirometric indices were only seen in patients with widespread tracheobronchial Kaposi's sarcoma, reflecting their extensive airways disease. All patients had reduced TLCo and Kco.Paradoxically, in those with widespread Kaposi's sarcoma the TLCo and Kco were relatively well preserved, perhaps reflecting the highly vascular nature of the tumour. Occult alveolar haemorrhage in six patients with widespread disease may also have influenced these results.

Follow up lung function testing in four patients who were not treated showed further reductions in TLCo and Kco. Surprisingly, despite deterioration of the chest radiograph in all four patients and bronchoscopic evidence of progression of tracheobroncial disease in three, only two patients had further reductions in $\mathrm{FEV}_{1}$ and FVC. All three patients who received chemotherapy had palliation of symptoms, yet in two patients the chest radiograph deteriorated and in all three the TLco fell. In one of these patients repeat bronchoscopy indicated that tracheobronchial disease had responded to treatment; yet $\mathrm{FEV}_{1}, \mathrm{FVC}$, and TLco had fallen. These results suggest that neither palliation of symptoms nor bronchoscopic improvement necessarily reflects the response of intrapulmonary Kaposi's sarcoma to chemotherapy.

In conclusion, pulmonary Kaposi's sarcoma produces abnormalities of TLCO even in patients with localised tracheobronchial disease; in patients with widespread disease airflow obstruction may occur. If the disease is untreated, progressive abnormalities are seen on the chest radiograph and in the results of pulmonary function tests. Even though chemotherapy palliates symptoms, some patients have progressive abnormalities of lung function. Reassessment of the extent of disease by bronchoscopy may not accurately reflect the response to chemotherapy.

We thank Jane Healing for help with the acquisition of data and Jane Catchpole for typing the manuscript. The Autolink lung function equipment was donated by CRUSAID.

1 Peters B, Beck EJ, Coleman DG, Wadsworth MJH, McGuiness $O$, Harris JRW, et al. Changing disease patterns in patients with AIDS in a referral centre in the patterns in patients with AIDS in a referral centre in the United Kin
302:203-7.

2 Zibrak JD, Silvestri RC, Costello P, Marlink R, Jensen WA Robins A, et al. Bronchoscopic and radiographic features of Kapsosi's sarcoma involving the respiratory tract. Chest 1986;90:476-9.

3 National Heart, Lung and Blood Workshop. Pulmonary complications of the acquired immunodeficiency syndrome. $N$ Engl J Med 1984;310:1682-8.

4 Garay SM, Belenko M, Fazzini E, Schinella R. Pulmonar manifestations of Kaposi's sarcoma. Chest 1987;91:39-43.

5 Gill PS, Akil B, Colletti P, Rarick M, Loureiro C, BernsteinSinger M, et al. Pulmonary Kapsoi's sarcoma: clinical findings and results of therapy. Am J Med 1989;87:57-61

Meduri GU, Stover DE, Lee M, Myskowski PL, Carvell JF, Zaman MB. Pulmonary Kaposi's sarcoma in the JF, Zaman MB. Pulmonary Kaposi's sarcoma in the acquired immunodeficiency syndrome. Am J Med 1986; 81:11-8.

7 Pitchenick AE, Fischl MA, Saldana MJ. Kaposi's sarcoma of the tracheobronchial tree. Clinical, bronchoscopic and pathological features. Chest 1985;87:122-4.

8 Hanson PV, Harcourt-Webster JN, Gazzard BG, Collins JV. Fibreoptic bronchoscopy in diagnosis of broncho- 
pulmonary Kaposi's sarcoma. Thorax 1987;42:269-71.

9 Miller RF, Kocjan G, Buckland J, Holton J, Malin A Semple SJG. Sputum induction for the diagnosis of pulmonary disease in HIV positive patients. $J$ Infec 1991;23:5-15.

10 Hughes-Davies L, Kocjan G, Spittle MF, Miller RF. Occult alveolar haemorrhage in bronchopulmonary Kaposi's sarcoma. J Clin Pathol 1992;45:536-7.

11 Shaw RJ, Roussak C, Forester SM, Harris JR, Pinching AJ, Mitchell DM. Lung function in patients infected with the human immunodeticiency virus with and without overt pneumonitis. Thorax 1988;43:436-40.

12 Fouret PJ, Touboul JL, Mayaud CM, Akoun GM, Roland J. Pulmonary Kaposi's sarcoma in patients with acquired immunodeficiency syndrome: a clinicopathological study. Thorax 1987;42:262-8.

13 Kaplan LD, Hopewell PC, Jaffe H, Goodman PC, Bottles K, Volberding PA. Kaposi's sarcoma involving the lung in patients with the acquired immunodeficiency syndrome. J Acq Imm Defic Synd 1988;1:23-30. 